\title{
Completely Integrable Models in Quasicrystals
}

\author{
V. E. Korepin \\ Leningrad Department of Steklov Mathematical Institute, Fontanka 27, \\ SU-191011 Leningrad, USSR
}

\begin{abstract}
The general method of construction of integrable dynamical models in quasicrystals is presented in the paper. It is illustrated on the example of the model of interacting spins for Penrose nonperiodic tiling of the plane. Another example constructed is the three dimensional model of interacting spins for icosahedral tiling of the three dimensional space. The bulk free energy is calculated for these models.
\end{abstract}

\section{Introduction}

The interest for nonperiodic tilings came first from problems of mathematical logics $[1,2]$. However, since the invention by Penrose of his well known aperiodic tilings of the plane [3-5], the motivations have changed to the study of the geometrical properties of these patterns. The important role in the study of these tilings belongs to J. Conway and de Bruijn [6]. A tiling is a covering of the whole plane by shifts of a finite number of polygons, which don't overlap, and there are no holes. The tiling is not periodic (there exists no shift that does not change the tiling). But it is quasiperiodic. It means that any finite part of the tiling appears infinitely many times in the whole tiling. Some of the tilings have additional symmetry. Penrose tiling has the axis of the fifth order, which is forbidden for the periodic tiling. Another kind of symmetry is deflation. The initial polygons can be cut into pieces, these pieces can be sewn in such way that the new tiling is similar to the initial one. Below we shall discuss the tiling of the plane by two rhombuses, see Fig. 1. The deflation for these rhombuses is depicted in Fig. 2. The inverse transformation is called inflation.

R. Ammann and Mackay [7] proposed a three dimensional generalisation of Penrose nonperiodic tiling. The three dimensional tiling has icosahedral symmetry [8] which is incompatible with a periodic lattice. The experimental discovery of icosahedral symmetry (a rapidly quenched alloy of $\mathscr{A}_{6} \mathscr{M}_{n}$ ) [9] played an important role. Its crystalline structure was explained by the Penrose nonperiodic tiling [10-12]. (It was the first example of a quasicrystal.) This discovery has 
aroused intense activity among theorists [13-21]. The interesting problem is to analyze dynamical systems in the quasicrystal [22-27].

The main result of this paper is the construction of exactly solvable models in quasicrystals. (Exactly solvable dynamical models are studied intensively in the literature, see [28-44] and references in these papers.) The general method of this paper is illustrated in two examples. The eight-vertex model is constructed for Penrose nonperiodic tiling of the plane. The eight-vertex model of interacting spins is equivalent to two interacting Ising models. In the three dimensional case the Zamolodchikov solution of tetrahedron equations is used to construct an exactly solvable model of interacting spins for icosahedral tiling of the three dimensional space.

In Sect. 2 Penrose nonperiodic tiling of the plane by two rhombuses is constructed in such a way, which permits us in Sect. 3 to construct a completely integrable model of interacting spins for this tiling. In Sect. 4 icosahedral tiling of the three dimensional space is constructed. Two rhombohedra are used instead of two rhombuses. Rhombohedron is a special case of parallelepiped. Section 5 is devoted to the Zamolodchikov model of interacting spins in three dimensions. The model is based on the solution of tetrahedron equations. In Sect. 6 the model of interacting spins is constructed for a three dimensional quasicrystal.

\section{Penrose Patterns}

Here we shall remind the reader of the construction of nonperiodic tiling of the plane by rhombuses, see Fig. 1. We shall denote this tiling by $\mathbb{Q}$. Each rhombus of the tiling can be obtained from a pair of rhombuses by translation and rotation. This pair is: the obtuse rhombus (angles $72^{\circ}$ and $108^{\circ}$ ) and the acute rhombus (angles $36^{\circ}$ and $144^{\circ}$ ), see Fig. 2. The length of each of the sides is equal to one. To describe an orientation of the rhombuses in the tiling it is convenient to introduce five unit vectors,

$$
\mathbf{e}_{J}=\exp \{2 \pi i J / 5\}, \quad J=0,1,2,3,4 .
$$

Here we used complex coordinates in the plane. Complex conjugate vectors are equal to $\mathbf{e}_{J}^{*}=\mathbf{e}_{-J}$; we use here formula (2.1) for $J=0,-1,-2,-3,-4$. It will be also useful to introduce five vectors $\mathbf{d}_{j}$, which are orthogonal to the vectors $\mathbf{e}_{J}$ :

$$
\mathbf{d}_{j}=\exp \left\{i \frac{\pi}{2}+i \frac{\pi}{5} j\right\}, \quad j=0,1,2,3,4 .
$$

The $J \leftrightarrow j$ correspondence is given by the following table (corresponding vectors are orthogonal):

$$
\mathbf{d}_{0} \leftrightarrow \mathbf{e}_{0}, \quad \mathbf{d}_{1} \leftrightarrow \mathbf{e}_{3}, \quad \mathbf{d}_{2} \leftrightarrow \mathbf{e}_{1}, \quad \mathbf{d}_{3} \leftrightarrow \mathbf{e}_{4}, \quad \mathbf{d}_{4} \leftrightarrow \mathbf{e}_{2} .
$$

All vectors $\mathbf{d}_{j}$ belong to the left half plane. Let us associate rhombus $r_{k j}$ to each pair of vectors $\mathbf{d}_{k}, \mathbf{d}_{j}(k>j)$. They are orthogonal to the edges of the rhombus $r_{k j}$, see Fig. 3. In this way the set of vectors (2.2) generates ten basic rhombuses, five obtuse ones and five acute ones (in different orientations). So we shall label each of basic rhombuses by a pair of numbers $j, k(4 \geqq k>j \geqq 0)$. All rhombuses of the tiling $\mathbb{Q}$ 


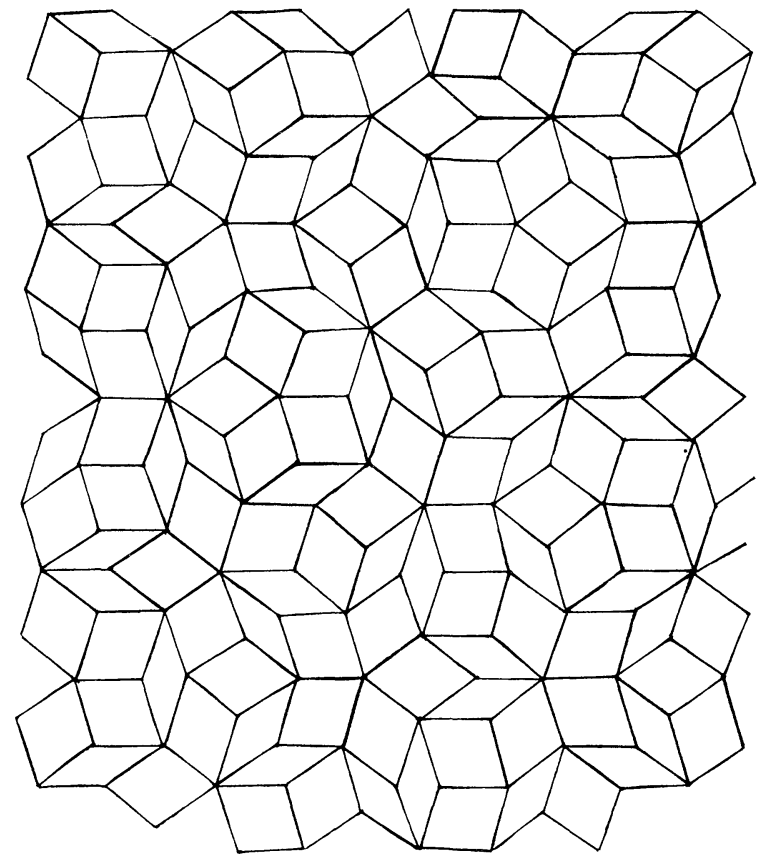

Fig. 1. Penrose nonperiodic tiling of the plane by two rhombuses
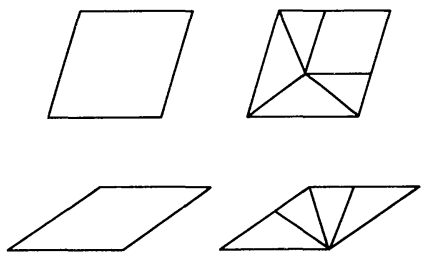

Fig. 2. Obtuse and acute rhombuses and their deflation

can be obtained from the basic ones using translations only. The relative frequency $\omega_{k j}$ of appearance of a given basic rhombus in the whole tiling is important. In the case when tiling has pentagonal symmetry [4-6] the frequencies of each acute rhombus are the same (we shall denote it by $\omega_{a}$ ) and the frequencies of all obtuse rhombuses are also the same $\left(\omega_{0}\right)$. They are equal to [3-6]:

$$
\omega_{0}=1 / 5 \tau, \quad \omega_{a}=1 / 5 \tau^{2} .
$$

Here $\tau$ is a golden ratio:

$$
\tau=\left(1+5^{1 / 2}\right) / 2 .
$$

(It should be noted that the plane can be tiled by translations of only one basic rhombus in the periodic way.) 
Now we shall give the construction of this tiling $\mathbb{Q}$ by means of the duality transformation [6]. It is very useful for construction of completely integrable models, see Sect. 3. First we shall construct the grid $\mathbb{G}$, and then by means of the duality transformation we shall construct nonperiodic tiling $\mathbb{Q}$. The grid $\mathbb{G}$ is constructed as follows. Let us consider the straight line $\ell_{J}$ orthogonal to the fixed vector $\mathbf{e}_{J}(2.1)$ :

$$
\ell_{J}\left(n_{J}\right)=\left\{z \in \mathbb{C} \mid \operatorname{Re}\left(z \mathbf{e}_{-J}\right)+\gamma_{J}=n_{J}\right\} .
$$

Here $z$ is the point of the complex plane, $\gamma_{J}$ is a real parameter and $n_{J}$ is an integer number. The straight line $\ell_{J}$ is directed along vector $\mathbf{d}_{j}$, see (2.2), (2.3). Let us consider now the equidistant set of parallel lines $\ell_{J}$. It means that $n_{J}$ takes all integer numbers from $-\infty$ to $+\infty$. Construct now such a system of straight lines for each of five vectors $\mathbf{e}_{J}(2.1)$. Parameters $\gamma_{J}$ should be chosen in such a way that no three straight lines $\ell_{J}$ intersect in one point. This set of straight lines will cut the plane into an infinite set of different polygons; we shall call them the faces of the grid. So we have constructed the grid $\mathbb{G}$.

Let us make the duality transformation. The vertices $\mathbf{v}$ of $\mathbb{Q}$ correspond to the faces of $\mathbb{G}$. The edges of $\mathbb{Q}$ correspond to the edges of $\mathbb{G}$ (they are orthogonal). The faces of $\mathbb{Q}$ correspond to the vertices of $\mathbb{G}$. To do the duality transformation it is convenient to associate five integer numbers $n_{J}$ with each face $\mathscr{F}$ of the grid:

$$
n_{J}(\mathscr{F})=\left[\operatorname{Re}\left(z \mathbf{e}_{-J}\right)+\gamma_{J}\right], \quad z \in \mathscr{F} .
$$

Square brackets here mean the integer part. [Roughly speaking $n_{J}$ are the numbers of the straight lines (2.6), which form the boundary of the face $\mathscr{F}$.] The vertex $\mathbf{v}$ of $\mathbb{Q}$, corresponding to $\mathscr{F}$, is given by:

$$
\mathbf{v}(\mathscr{F})=\sum_{J=0}^{4} \mathbf{e}_{J} n_{J}(\mathscr{F})
$$

In this way all the vertices are constructed. Let us construct the edges of $\mathbb{Q}$. We shall connect some of vertices by the segments of straight lines. Consider two adjacent faces of $\mathbb{G}$ (they have a common edge) and connect the vertices $\mathbf{v}$ corresponding to these two faces by a vector. One can show that this vector is one of five vectors (2.1). So each edge of $\mathbb{Q}$ can be obtained by shifts from (2.1). The faces of $\mathbb{Q}$ correspond to vertices of $\mathbb{G}$. To see this consider four faces of $\mathbb{G}$, which have a common vertex. Four vertices of $\mathbb{Q}$, corresponding to these faces are the vertices of the face of $\mathbb{Q}$. This face is the convex hull of its vertices. Faces of $\mathbb{Q}$ are the ten basic rhombuses mentioned above. So we have constructed the tiling $\mathbb{Q}$.

It should be mentioned that the duality transformation is a general method for construction of nonperiodic tilings $[6,8,13,16,21]$. (There exist a lot of different nonperiodic tilings.) For each of these tilings the completely integrable spin model can be constructed, see the next section.

\section{Exactly Solvable Spin Model in Two Dimensional Quasicrystal}

Here we shall construct an eight-vertex spin model for the Penrose tiling of the plane, described in Sect. 2. Spins $\sigma$ are associated with the vertices $\mathbf{v}$ of the tiling $\mathbb{Q}$. 


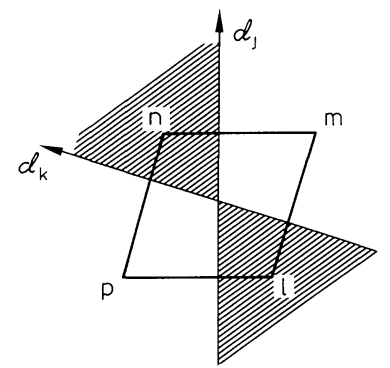

Fig. 3. This is basic rhombus $r_{k j}$. Vectors $\mathbf{d}_{k}, \mathbf{d}_{j}$ are orthogonal to its edges

Each spin has two values \pm 1 . Spins interact round a face of $\mathbb{Q}$. (All faces are rhombuses.) The Hamiltonian $H$ of the model is equal to

$$
\frac{-H}{T}=\sum_{\text {faces }}\left[P_{k j}^{\prime} \sigma_{\ell} \sigma_{n}+P_{k j} \sigma_{p} \sigma_{m}+P^{\prime \prime} \sigma_{\ell} \sigma_{m} \sigma_{n} \sigma_{p}\right]
$$

Here $T$ is the temperature. The sum is over all the faces of $\mathbb{Q}$. The expression in the square brackets is the contribution of the given rhombus $r_{k j}$ to the Hamiltonian. Spins $\sigma_{\ell}, \sigma_{n}, \sigma_{m}, \sigma_{p}$ are situated in the vertices of the rhombus, see Fig. 3 . The values $P_{k j}, P_{k j}^{\prime}$, and $P^{\prime \prime}$ are dynamical coefficients. Let us consider the vectors $\mathbf{d}_{j}(2.2)$; they belong to the left half plane. First consider the pair of vectors $\mathbf{d}_{k}, \mathbf{d}_{j}(k>j)$ corresponding to the given basic rhombus $r_{k j}$, see Fig. 3. (They are orthogonal to its edges.) Shade the sector between these two vectors (and the opposite sector). The pair of vertices of rhombus $r_{k j}$ belong to the shaded sectors $\left(\sigma_{n}, \sigma_{\ell}\right)$, another pair belong to unshaded sectors $\left(\sigma_{p}, \sigma_{m}\right)$. The interaction of spins in the shaded sectors is given by the coefficients $P_{k j}^{\prime}$, the interaction of the spins in the unshaded sectors are given by coefficients $P_{k j}$. Coefficients $P_{k j}$ and $P_{k j}^{\prime}$ are orientational dependent. There are twenty one dynamical coefficients. The object of statistical mechanics is to calculate the partition function:

$$
Z=\sum_{\{\sigma\}} \exp \{-H / T\}
$$

In the thermodynamical limit the free energy $F=-T \ln Z$ is proportional to the number of rhombuses $N$. The bulk free energy $\mathfrak{f}$ is of interest:

$$
\mathfrak{f}=\lim (F / N) \text {. }
$$

It would be highly desirable to solve the model for any choice of dynamical coefficients, but the author knows of no way to do this. What can be done is to calculate $\mathfrak{f}$, if dynamical coefficients depend (in some special way) on six parameters. The corresponding parametrisation can be written in terms of elliptic functions. Their modulus $k$ is the first parameter. Each unit vector $\mathbf{d}_{j}(2.2)$ is associated with an independent real parameter $\alpha_{j}(j=0,1,2,3,4)$. We shall call $\alpha_{j}$ spectral parameters. They form an increasing sequence $\alpha_{k}>\alpha_{j}$, if $k>j$. The last parameter is the coupling constant $\lambda$. Let us associate the values

$$
\alpha_{k j}^{\prime} \equiv \alpha_{k}-\alpha_{j}, \quad \alpha_{k j} \equiv \lambda+\alpha_{j}-\alpha_{k}, \quad k>j
$$


with each basic rhombus. These parameters must satisfy the following inequalities:

$$
0<\alpha_{k j}, \alpha_{k j}^{\prime}<\lambda<2 \mathscr{K}^{\prime}, \quad k>j .
$$

The values $\mathscr{K}$ and $\mathscr{K}^{\prime}$ are the complete elliptic integrals of the first kind of moduli $k$ and $k^{\prime}=\left(1-k^{2}\right)^{1 / 2}$ respectively. Dynamical coefficients are expressed in terms of six independent parameters by the following formulas:

$$
\begin{aligned}
& 2 i P_{k j}^{\prime}=\operatorname{am}\left\{i\left(\mathscr{K}^{\prime}-\alpha_{k j}^{\prime}\right)\right\}, \\
& 2 i P_{k j}=\operatorname{am}\left\{i\left(\mathscr{K}^{\prime}-\alpha_{k j}\right)\right\}, \\
& 2 i P^{\prime \prime}=-\operatorname{am}\left\{i\left(\mathscr{K}^{\prime}-\lambda\right)\right\} .
\end{aligned}
$$

The values $\alpha_{k j}^{\prime}$ fix the interaction of spins in the shaded vertices of the rhombus (Fig. 3), and $\alpha_{k j}$ fix the interaction of spins in the unshaded vertices of the rhombus. In this way the exactly solvable spin model is constructed.

The model can be solved in the following way. First one must reformulate the model on the grid $\mathbb{G}$ dual to the quasiperiodic tiling $\mathbb{Q}$, see Sect. 2. Now spins $\sigma$ are associated with the faces of the grid. Spins interact round a vertex of the grid. Each straight line $\ell_{J}(2.6)$ is associated with the spectral parameter $\alpha_{j}$, see (2.3). The value of the spectral parameter is the same for the parallel straight lines. So this model is equivalent to the special case of the eight-vertex model on an arbitrary planar lattice [33]. It permits us to solve the model. The bulk free energy $\mathfrak{f}$ is equal to:

$$
\mathfrak{f}=\sum_{4 \geqq k>j \geqq 0} \omega_{k j} \mathfrak{f}_{0}\left(P_{k j}, P_{k j}^{\prime}, P^{\prime \prime}\right) \text {. }
$$

The sum is over ten basic rhombuses, $\omega_{k j}$ are relative frequencies $(2.4), \tilde{f}_{0}$ is the bulk free energy for the periodic case. For a periodic tiling of the plane by the shifts of one of the basic rhombuses the spin model is defined in a similar way. It is equivalent to the standard eight-vertex model, the exact solution of which is presented in detail in the book [28]. The bulk free energy of this model is denoted by $\tilde{f}_{0}$. Solution of the periodic model was imbedded in the frame of quantum inverse scattering method in the paper [32]. The periodic model depends only on three dynamical coefficients $P, P^{\prime}, P^{\prime \prime}$. Partition function $Z$ has a symmetry (see the Appendix), which permits us to consider dynamical coefficients only in the region:

$$
\exp \left(P+P^{\prime}+P^{\prime \prime}\right) \geqq \exp \left(P-P^{\prime}-P^{\prime \prime}\right)+\exp \left(-P+P^{\prime}-P^{\prime \prime}\right)+\exp \left(-P-P^{\prime}+P^{\prime \prime}\right) .
$$

In this region the bulk free energy $\mathfrak{f}_{0}$ in the periodic case is equal to:

$$
-\mathfrak{f}_{0}\left(P, P^{\prime}, P^{\prime \prime}\right) / T=P+P^{\prime}+P^{\prime \prime}+\sum_{n=1}^{\infty} \frac{x^{-n}\left(x^{2 n}-q^{n}\right)^{2}\left(x^{n}+x^{-n}-z^{n}-z^{-n}\right)}{n\left(1-q^{2 n}\right)\left(1+x^{2 n}\right)} .
$$

Here

$$
\begin{gathered}
q=\exp \left\{-2 \pi \mathscr{K}^{\prime} / \mathscr{K}\right\}, \quad x=\exp \{-\pi \lambda / 2 \mathscr{K}\}, \\
z=\exp \left\{-\pi\left(\alpha^{\prime}-\alpha\right) / 2 \mathscr{K}\right\} .
\end{gathered}
$$




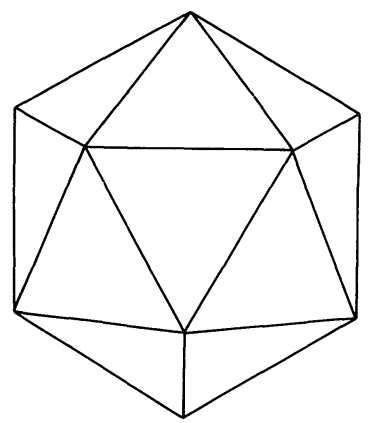

Fig. 4. Icosahedron generates crystalline structure of $\mathscr{A} \ell \mathscr{M} n$ alloy

The local magnetization and polarization are equal to

$$
\begin{gathered}
\left\langle\sigma_{\ell}\right\rangle=\prod_{n=1}^{\infty}\left(\frac{1-x^{4 n-2}}{1+x^{4 n-2}}\right), \\
\left\langle\sigma_{\ell} \sigma_{m}\right\rangle=\prod_{n=1}^{\infty}\left(\frac{1+q^{n}}{1-q^{n}}\right)^{2}\left(\frac{1-x^{2 n}}{1+x^{2 n}}\right)^{2} .
\end{gathered}
$$

So we have solved the spin model in the two dimensional quasicrystal. In the case $P^{\prime \prime}=0$, (3.1), (3.6) the model is equivalent to two Ising Hamiltonians for the quasicrystal. The Ising model is equivalent to free fermions. In the case $P^{\prime \prime}=0$ correlation functions can be calculated, see [33]. For correlation functions of interacting fermions see for example [34].

To conclude, one has to emphasize that we have presented a general method of construction of integrable models in the quasicrystals. A quasicrystal can be obtained by means of the duality transformation from the grid $[6,8,13,16,21]$. The eight-vertex model can be solved on any grid [33]. It is clear that not only the eight-vertex model but any solution of the Yang-Baxter equations [28-31] can be used for construction of the integrable models in the quasicrystals.

\section{Icosahedral Tiling of $\mathbb{R}^{3}$}

The three dimensional case is treated similar to the two dimensional one considered above. The most interesting quasicrystal has icosahedral symmetry [7, $8,10-12,15,17]$. There exist different icosahedral tilings of $\mathbb{R}^{3}$.

We shall illustrate below the quasiperiodic tiling of $\mathbb{R}^{3}$ by means of two rhombohedra $[7,8,15,17]$. Rhombohedron is a special case of parallelepiped; all its faces are identical to the same rhombus. The ratio of diagonals of this rhombus is equal to $\tau(2.5)$. First we shall construct the grid $\mathbb{G}$, and then (by-means of the duality transformation) the quasicrystal $\mathbb{Q}$. Consider an icosahedron, see Fig. 4. It has six axes of the fifth order. Let us consider six unit vectors

$$
\mathbf{e}_{j}, \quad j=1,2,3,4,5,6,
$$


directed along these six axes. Regard the plane $p_{j}$ orthogonal to the vector $\mathbf{e}_{j}$,

$$
p_{j}\left(n_{j}\right)=\left\{\mathbf{x} \mid\left(\mathbf{e}_{j} \mathbf{x}\right)+\gamma_{j}=n_{j}\right\} .
$$

Here $\mathbf{x} \in \mathbb{R}^{3}$ and $\gamma_{j}$ are six independent real parameters, brackets denote the scalar product, $n_{j}$ is the integer number (corresponding to this plane). Consider now the system of parallel, equidistant planes orthogonal to the vector $\mathbf{e}_{j}$. Each plane of this system is given by the formula (4.2) and the variable $n_{j}$ takes all integer values $-\infty<n_{j}<+\infty$. There are six different systems of planes of this kind, corresponding to each $\mathbf{e}_{j}$ (4.1). This system of planes divides $\mathbb{R}^{3}$ into the set of polyhedrons; they are cells of the grid $\mathbb{G}$. Parameters $\gamma_{j}$ should be chosen in such a way that no four planes have a common point. So we have constructed grid $\mathbb{G}$. To make the duality transformation let us associate six integer numbers $n_{j}$ with each cell of the grid. Let $\mathbf{x}$ belong to some cell $c$ of the grid. Integer numbers are given by the following formula:

$$
n_{j}(c)=\left[\left(\mathbf{x e}_{j}\right)+\gamma_{j}\right], \quad \mathbf{x} \in c .
$$

(Roughly speaking these $n_{j}$ are the integer numbers corresponding to the planes bounding the cell.) Square brackets mean integer parts. The vertices $\mathbf{v}$ of $\mathbb{Q}$ correspond to the cell of $\mathbb{G}$; they are given by the formula

$$
\mathbf{v}(c)=\sum_{j=1}^{6} \mathbf{e}_{j} n_{j}(c)
$$

The vertices corresponding to the adjacent cells of the grid differ by one of vectors $\mathbf{e}_{j}$ (4.1) $\left(\mathbf{v}_{1}=\mathbf{v}_{2}+\mathbf{e}_{j}\right)$. Let us connect these vertices by the segments of straight lines (vectors). It will be the edges of $\mathbb{Q}$. The faces of $\mathbb{Q}$ are constructed as follows. Let us regard four vertices $\mathbf{v}_{1}, \mathbf{v}_{2}, \mathbf{v}_{3}, \mathbf{v}_{4}$ of $\mathbb{Q}$ corresponding to such four cells of the grid, which have a common edge. They are connected by four edges, which form the boundary of rhombus. The rhombus spanned on this boundary is the face of $\mathbb{Q}$. The cell of the quasicrystal $\mathbb{Q}$ corresponds to the vertex of the grid $\mathbb{G}$. The cells of $\mathbb{Q}$ are rhombohedra $\mathbb{F}$. The basic rhombohedron $\mathbb{F}_{i k j}$ is generated by three of six vectors $\mathbf{e}_{j}(4.1)$,

$$
\mathbb{F}_{i k j}=\lambda_{i} \mathbf{e}_{i}+\lambda_{k} \mathbf{e}_{k}+\lambda_{j} \mathbf{e}_{j}, \quad 0 \leqq \lambda_{i, k, j}<1, j>k>i .
$$

Here $\lambda_{j}$ are real numbers. We shall label each basic rhombohedron by three numbers $i, k, j$ (the numbers of vectors e generating rhombohedron). There are twenty $\left(C_{6}^{3}\right)$ basic rhombohedra. Each rhombohedron of the tiling $\mathbb{Q}$ can be obtained from the basic ones by translations. One can show that ten of the basic rhombohedra can be obtained from one standard rhombohedron by means of rotations. We shall call this standard rhombohedron the acute rhombohedron. It is generated (4.5) by the three following unit vectors:

$$
\begin{gathered}
\mathbf{e}_{1}=\alpha(0,1, \tau), \quad \mathbf{e}_{2}=\alpha(-\tau, 0,-1), \quad \mathbf{e}_{3}=\alpha(\tau, 0,-1), \\
\alpha=\left(1+\tau^{2}\right)^{1 / 2}, \quad \tau=\left(1+5^{1 / 2}\right) / 2 .
\end{gathered}
$$

Ten other basic rhombohedra can be obtained from the obtuse rhombohedron by rotations. It is generated (4.5) by the three following unit vectors:

$$
\mathbf{e}_{4}=\alpha(0,-1, \tau), \quad \mathbf{e}_{5}=(\tau, 0,1) \alpha, \quad \mathbf{e}_{6}=\alpha(0,1, \tau) .
$$


The relative frequency $\omega_{i k j}$ of the appearance of each basic rhombohedron can be calculated [15]. It is the same for all acute rhombohedra and equal to

$$
\omega_{a}=1 / 10 \tau^{2} .
$$

The relative frequency $\omega_{i k j}$ for all obtuse rhombohedra is equal to:

$$
\omega_{0}=1 / 10 \tau \text {. }
$$

At last, one should emphasize that there are many different nonperiodic tilings (quasicrystals). The icosahedral symmetry is not necessary for them. The method of the dual transformation from the grid is a general method for constructing quasicrystals $[6,13,8,16,21]$. In Sect. 6 , we shall construct a completely integrable spin system for an arbitrary quasicrystal.

\section{Zamolodchikov Model}

Zamolodchikov constructed the model of interacting spins in three dimensions $[35,36]$. The Boltzmann weights for this model satisfy tetrahedron equations; this is the reason why the model is exactly solvable [35-37]. The bulk free energy of the model was evaluated by Baxter in $[38,39]$. To prove that all tetrahedron equations are satisfied, Baxter reformulated the Zamolodchikov model on the dual lattice [40]. We shall use this formulation. Let us consider a standard cubic lattice $\mathscr{L}$ in three dimensional space. Spins $\sigma$ are associated with the vertices of the lattice. Each spin is free to take two values \pm 1 . They interact round a cube. The Boltzmann weight is associated with each unit cube. The disposition of spins in the vertices of the unit cube is shown in Fig. 5. We shall denote the Boltzmann weight associated with this cube by:

$$
W(a|e, f, g| b, c, d \mid h) .
$$

This weight depends not only on the values of spins $a, b, c, d, f, g, h, e$, but also on three real parameters $\theta_{12}, \theta_{23}, \theta_{13}$ (angles). It can be written as follows:

$$
W\left(a|e, f, g| b, c, d \mid h ; \theta_{23}, \theta_{13}, \theta_{12}\right) .
$$

To define these parameters let us introduce three unit (three dimensional) vectors $\mathbf{n}_{1}, \mathbf{n}_{2}, \mathbf{n}_{3}$. They do not necessarily coincide with three unit vectors generating the

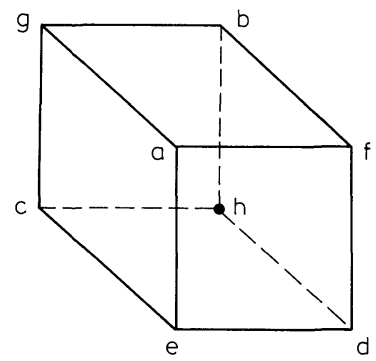

Fig. 5. Arrangement of the spins $a, \ldots, h$ on the corner sites of a cube 
three dimensional lattice. The vectors $\mathbf{n}_{1}, \mathbf{n}_{2}, \mathbf{n}_{3}$ are similar to the spectral parameters; they define the angles $\theta$ :

$$
\left(\mathbf{n}_{1} \mathbf{n}_{2}\right)=\cos \theta_{12}, \quad\left(\mathbf{n}_{1} \mathbf{n}_{3}\right)=\cos \theta_{13}, \quad\left(\mathbf{n}_{2} \mathbf{n}_{3}\right)=\cos \theta_{23} .
$$

To write down the explicit expression of $W$, let us introduce spherical excesses:

$$
2 \alpha_{0}=\theta_{12}+\theta_{13}+\theta_{23}-\pi, \quad \alpha_{i}=\theta_{j k}-\alpha_{0} .
$$

Here $i, j, k$ are a permutation of $1,2,3$. Further define

$$
\begin{gathered}
t_{\ell}=\left\{\tan \left(\alpha_{l} / 2\right)\right\}^{1 / 2}, \quad s_{\ell}=\left\{\sin \left(\alpha_{l} / 2\right)\right\}^{1 / 2}, \\
c_{\ell}=\left\{\cos \left(\alpha_{l} / 2\right)\right\}^{1 / 2}, \quad \ell=0,1,2,3,
\end{gathered}
$$

and

$$
\begin{gathered}
P_{0}=1, \quad Q_{0}=t_{0} t_{1} t_{2} t_{3}, \quad R_{0}=s_{0} /\left(c_{1} c_{2} c_{3}\right), \\
P_{i}=t_{j} t_{k}, \quad Q_{i}=t_{0} t_{i}, \quad R_{i}=s_{i} /\left(c_{0} c_{j} c_{k}\right) .
\end{gathered}
$$

The Boltzmann weight $W(5.2)$ has the values given in the following Table 1.

\section{Table 1}

\begin{tabular}{llll}
\hline abeh & $a c f h$ & $a d g h$ & $W\left(a|e, f, g| b, c, d \mid h ; \theta_{23}, \theta_{13}, \theta_{12}\right)$ \\
\hline+ & + & + & $P_{0}-a b c d Q_{0}$ \\
- & + & + & $R_{1}$ \\
+ & - & + & $R_{2}$ \\
+ & + & - & $R_{3}$ \\
+ & - & - & $a b P_{1}+c d Q_{1}$ \\
- & + & - & $a c P_{2}+b d Q_{2}$ \\
- & - & + & $a d P_{3}+b c Q_{3}$ \\
- & - & - & $R_{0}$
\end{tabular}

The partition function of the model is defined as:

$$
Z=\sum_{\{\sigma\}} \prod_{\text {cubes }} W(a|e, f, g| b, c, d \mid h) .
$$

The bulk free energy $\mathfrak{f}_{0}\left(\theta_{23}, \theta_{13}, \theta_{12}\right)$ of the model is equal to [39]:

$$
\begin{aligned}
& -\mathfrak{f}_{0}\left(\theta_{23}, \theta_{13}, \theta_{12}\right) / k_{B} T \\
& =-\ln \left(c_{0} c_{1} c_{2} c_{3}\right)+\Phi(\pi-s)+\Phi\left(s-a_{1}\right)+\Phi\left(s-a_{2}\right)+\Phi\left(s-a_{3}\right) \\
& \quad+\frac{1}{2 \pi} \sum_{i=1}^{3}\left\{a_{i} \ln \sin \left(\theta_{j k} / 2\right)+\left(\pi-a_{i}\right) \ln \cos \left(\theta_{j k} / 2\right)\right\} .
\end{aligned}
$$

Here $k_{B}$ is Boltzmann's constant, $T$ is the temperature. The values $a_{1}, a_{2}, a_{3}$ are the three sides of the spherical triangle opposite to the angles $\theta_{23}, \theta_{13}, \theta_{12}$. The perimeter of this triangle will be denoted $2 s$ :

$$
2 s=a_{1}+a_{2}+a_{3} .
$$


Here $i, j, k$ are permutations of $1,2,3$. The polylogarithm function $\Phi(x)$ is defined as:

$$
\Phi(x)=\sum_{m=1}^{\infty} \frac{\sin (2 m x)}{2 \pi m^{2}} .
$$

One should mention that the partition function (5.8) is $Z$-invariant [31], due to the fact that weights $W$ satisfy tetrahedron equations.

At last let us formulate the model on the dual lattice and obtain the original formulation by Zamolodchikov. Consider the dual lattice $\mathscr{L}_{D}$ to the initial cubic lattice $\mathscr{L}$. It is also cubic, its vertices are situated in the centers of the unit cubes of the initial lattice $\mathscr{L}$. So the spins are situated now in the cubes of $\mathscr{L}_{D}$, and Boltzmann weights are associated with the vertices of $\mathscr{L}_{D}$. Make now the following transformation. Adjacent spins are separated by faces of $\mathscr{L}_{D}$. Let us define the spin on the face of $\mathscr{L}_{D}$ to be equal to the product of spins of the unit cubes, which are separated by this face. These are Zamolodchikov spins. The Boltzmann weight (associated with the vertex of $\mathscr{L}_{D}$ ) is denoted by

$$
\bigotimes \begin{aligned}
& i_{1} k_{1} i_{1}^{\prime} k_{1}^{\prime} \\
& i_{2} k_{2} i_{2}^{\prime} k_{2}^{\prime}\left(\theta_{23}, \theta_{13}, \theta_{12}\right) \text {. } \\
& i_{3} k_{3} i_{3}^{\prime} k_{3}^{\prime}
\end{aligned}
$$

Here $i$ and $k$ are the values of the spins on the three planes, intersecting in the vertex, see Fig. 6) Finally, $S$ and $W$ are related by the equality:

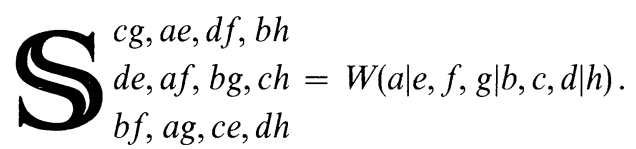

The arguments are given in [45] that the Zamolodchikov model is critical.

It should be mentioned that (similar to the two dimensional case) the Zamolodchikov model can be formulated on arbitrary set of planes (no four planes have a common point). The partition function will be $Z$ invariant. There exists also another exactly solvable three dimensional model [41]. It is equivalent to free fermions. It also can be used for construction of exactly solvable models in quasicrystals.

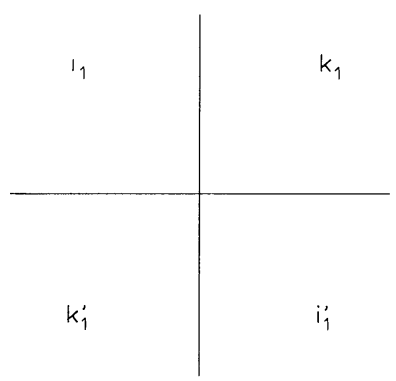

Fig. 6. The Boltzmann weight $\mathbf{S}$ of the Zamolodchikov model is associated with intersection of three planes. Spins $i, k$ are associated with the faces of the lattice $\mathscr{L}_{D}$. First plane intersected by two others is depicted 


\section{Exactly Solvable Spin Model for Three Dimensional Quasicrystal}

The three dimensional case is treated similar to the two dimensional one constructed in Sect. 3. Let us consider icosahedral tiling of $\mathbb{R}^{3}$, see Sect. 4. We shall construct the spin model for this quasicrystal $\mathbb{Q}$. Spins are situated in the vertices of $\mathbb{Q}$. Spins $\sigma$ are free to take two values \pm 1 . They interact round a rhombohedron. The Boltzmann weight $W$ associated with a rhombohedron is:

$$
W(a|e, f, g| b, c, d \mid h) .
$$

Spins $a, b, c, d, e, f, g, h$ are situated in the vertices of the rhombohedron in the same way as it was in the case of a cube, see Fig. 5. The weights $W$ depend also on spectral parameters. To introduce these parameters let us associate the unit vector $\mathbf{n}_{j}$ with each of the six vectors $\mathbf{e}_{j}$, generating the quasicrystal (4.1). Vector $\mathbf{n}_{j}$ does not necessarity coincide with $\mathbf{e}_{j}$; it plays the role of spectral parameter. The Boltzmann weight $W$, which is associated with the rhombohedron $\mathbb{F}_{i, j, k}$ [it is generated by three vectors $\left.\mathbf{e}_{i}, \mathbf{e}_{j}, \mathbf{e}_{k}(4.5)\right]$ depends on three angles $\theta_{j k}, \theta_{i k}, \theta_{i j}$ :

$$
\left(\mathbf{n}_{j} \mathbf{n}_{k}\right)=\cos \theta_{j k}, \quad\left(\mathbf{n}_{i} \mathbf{n}_{k}\right)=\cos \theta_{i k}, \quad\left(\mathbf{n}_{i} \mathbf{n}_{j}\right)=\cos \theta_{i j} .
$$

We shall denote the Boltzmann weight by

$$
W\left(a|e, f, g| b, c, d \mid h ; \theta_{j k}, \theta_{i k}, \theta_{i j}\right) \text {. }
$$

The explicit expression for $W$ is given by the table (5.7) (after replacement of $\theta_{23} \rightarrow \theta_{j k}, \theta_{13} \rightarrow \theta_{i k}, \theta_{12} \rightarrow \theta_{i j}$ ). The partition function of the model is defined as

$$
Z=\sum_{\{\sigma\}} \prod_{\text {rhombohedra }} W(a|e, f, g| b, c, d \mid h) .
$$

The partition function $Z$ depends on 9 angle parameters (scalar products of six unit vectors $\mathbf{n}$ ). The partition function is $Z$-invariant due to the fact that weights $W$ satisfy tetrahedron equations. It means that the model can be solved similar to the two dimensional case. One must reformulate the model on the grid dual to the quasicrystal. It will be the Zamolodchikov model on the arbitrary set of planes. Using the "unitarization hypothesis" $[42-44,28]$ one obtains for the bulk free energy $\mathfrak{f}$ :

$$
\mathfrak{f}=\sum_{6 \geqq j>k>i \geqq 1} \omega_{i k j} \mathfrak{f}_{0}\left(\theta_{j k}, \theta_{i k}, \theta_{i j}\right) .
$$

It is the sum of twenty terms; each of them corresponds to one of basic rhombohedron (4.5), $\omega_{i k j}$ being the relative frequency of appearance of a given rhombohedron, see (4.8), (4.9); $\mathfrak{\mathfrak { f }}_{0}$ is the bulk free energy for the periodic case, see (5.9). For the periodic tiling of $\mathbb{R}^{3}$ by means of translations of one of the basic rhombohedra the spin model is defined in a similar way. It is equivalent to the standard Zamolodchikov model, see Sect. 5.

The completely integrable spin model can be constructed in a similar way for an arbitrary quasicrystal. 


\section{Conclusion}

The general method of construction of integrable dynamical models in quasicrystals is presented in the paper. The properties of these models are close to properties of similar models in the periodic case, as distinct from properties of dynamical models in quasicrystals discussed before in the literature.

\section{Appendix. Symmetry of Partition Function}

Let us consider the eight-vertex spin model for two dimensional periodic lattice. The Boltzmann weight can be written in the form

$$
w=\exp \left\{P \sigma_{m} \sigma_{p}+P^{\prime} \sigma_{\ell} \sigma_{n}+P^{\prime \prime} \sigma_{\ell} \sigma_{m} \sigma_{n} \sigma_{p}\right\} .
$$

It takes the following values $a, b, c, d$ :

$$
\begin{aligned}
a, b, c, d= & \exp \left\{P-P^{\prime}-P^{\prime \prime}\right\}, \exp \left\{-P+P^{\prime}-P^{\prime \prime}\right\}, \exp \left\{P+P^{\prime}+P^{\prime \prime}\right\}, \\
& \exp \left\{-P-P^{\prime}+P^{\prime \prime}\right\} .
\end{aligned}
$$

The partition function has the following symmetry property [28]:

$$
\begin{gathered}
Z_{0}\left(\left\{a^{*}, b^{*}, c^{*}, d^{*}\right\}\right)=Z_{0}(\{a, b, c, d\}), \\
a^{*}=(a-b+c-d) / 2, \quad b^{*}=(-a+b+c-d) / 2, \\
c^{*}=(a+b+c+d) / 2, \quad d^{*}=(-a-b+c+d) / 2 .
\end{gathered}
$$

So it is sufficient to consider the model in the region (3.8).

Acknowledgements. I want to thank R. J. Baxter, A. B. Zamolodchikov, V. V. Bazhanov, and A. G. Izergin for useful discussions. I am grateful to my wife for help in preparing the manuscript.

\section{References}

1. Wang, H.: Games, logic, and computers. Sci. Am. 216, 98-107 (1965)

2. Robinson, R.M.: Undecidability and nonperiodicity for tilings of the plane. Invent. Math. 12, 177-209 (1971)

3. Penrose, R.: The role of aestetics in pure and applied mathematical research. Bull. Inst. Math. Appl. 10, 266-271 (1974)

4. Penrose, R.: Pentaplexity. Math. Intell. 2, 32-37 (1979)

5. Gardner, M.: Mathematical games. Sci. Am. 236, 110-121 (1977)

6. de Bruijn, N.G.: Algebraic theory of Penrose's nonperiodic tilings of the plane. Proc. K. Ned. Akad. Wet. Ser. A 43, 39-66 (1981)

7. Mackay, A.L.: De nive quinquangula. Kristallografiya [Sov. Phys. Crystallogr.] 26, 910 (1981)

8. Kramer, P., Neri, R.: On periodic and non-periodic space fillings of $\mathbb{E}^{m}$ obtained by projection. Acta Crystallogr. Sec. A 40, 580-587 (1984)

9. Shechtman, D., Blech, I., Gratias, D., Cahn, J.W.: Metallic phase with long-range orientational order and no translational symmetry. Phys. Rev. Lett. 53, 1951-1953 (1984)

10. Levine, D., Steinhardt, P.J.: Quasicrystals: a new class of ordered structures. Phys. Rev. Lett. 53, 2477-2480 (1984)

11. Kalugin, P.A., Kitayev, A.Yu., Levitov, L.S.: 6-dimensional properties of $\mathscr{A} \mathscr{\ell} \mathscr{U n}_{n}$ alloy. J. Phys. Lett. 46, L-601-607 (1985) 
12. Kalugin, P.A., Kitayev, A.Yu., Levitov, L.S.: $\mathscr{A} \ell \mathscr{M}_{n}$-six dimensional crystal. Pisma v ZETF (Sov.) 41, 119-121 (1986)

13. Socolar, J.E.S., Steinhardt, P.J., Levine, D.: Quasicrystals with arbitrary orientational symmetry. Phys. Rev. B 28, 5547-5550 (1985)

14. Elser, V., Henley, L.: Crystal and quasicrystal structures in $\mathscr{A} \ell-\mathscr{M}_{n}$-alloys. Phys. Rev. Lett. 55, 2883-2886 (1985)

15. Katz, A., Duneau, M.: Quasiperiodic patterns and icosahedral symmetry. J. Phys. 47, 181-196 (1986)

16. Gälhler, F., Rhyner, J.: Equivalence of generalised grid and projection method for the construction of quasiperiodic tilings. J. Phys. A (Math. Gen.) 19, 267-277 (1986)

17. Elser, V.: The diffraction pattern of projected structures. Acta Crystallographica 42 A, 36-43 (1986)

18. Rivier, N., Sadoc, J.F.: Hierarchy and disorder in noncrystalline structures. Preprint. London: Imperial College 1986

19. Levine, D., Steinhardt, P.J.: Quasicrystals, I. Phys. Rev. B 34, 596-616 (1986);

Socolar, J.E.S., Steinhardt, P.J.: Quasicrystals, II. Phys. Rev. B 34, 617-647 (1986)

20. Bak, P.: Symmetry, Stability, and elastic properties of icosahedral incommensurate crystals. Phys. Rev. B 32, 5764 (1985)

21. Korepin, V.E.: Quasiperiodic tilings and quasicrystals. Zap. Nauch. Semin. LOMI (Sov.) 155, 116-135 (1986)

22. Kohmoto, M., Kadanoff, L.P., Tang, C.: Phys. Rev. Lett. 50, 1870 (1983)

23. Ostlund, S., Pandit, R., Rand, D., Schellnhuber, H.J., Siggia, E.: One-dimensional Schrödinger equation with an almost periodic potential. Phys. Rev. Lett. 50, 1873 (1983)

24. Kalugin, P.A., Kitayev, A.Yu., Levitov, L.S.: Electronic spectrum of one-dimensional quasicrystal. Preprint. Moscow: L.D. Landau Institut 1986

25. Sutherland, B.: A self-similar ground state wave function for electrons on a two-dimensional Penrose lattice. Preprint. Salt Lake City: University of Utah 1986

26. Luck, J.M., Nieuwenhuzen, T.M.: A soluble quasi-crystalline magnetic model: The $X Y$ quantum spin chain. Europhys. Lett. 2, 257-266 (1986)

27. Korepin, V.E.: Eight-vertex model of the quasicrystal. Phys. Lett. A 118, 285-286 (1986)

28. Baxter, R.J.: Exactly solved models in statistical mechanics. New York, London: Academic Press 1982

29. Faddeev, L.D.: Quantum completely integrable models of field theory. Sov. Sci. Rev. Math. Phys. C 1, 107-160 (1981)

30. Izergin, A.G., Korepin, V.E.: Quantum inverse scattering method. Fizika ehlementarnyhh chastits I atomnogo yadra (Sov. J. Elem. Part. Nucl.) 13, 207-223 (1982)

31. Lecture Notes in Physics, No. 242. Shastry, B.S., Jha, S.S., Singh, V.(Hrsg.). Berlin, Heidelberg, New York: Springer 1985

32. Takhtajan, L.A., Faddeev, L.D.: Quantum inverse scattering method and XYZ Heisenberg model. Usp. Mat. Nauk. (Sov.) 34, 13-63 (1979)

33. Baxter, R.J.: Solvable eight-vertex model on an arbitrary planar lattice. Phil. Trans. R. Soc. Lond. 289, 315-346 (1978)

34. Izergin, A.G., Korepin, V.E.: Critical exponents in the Heisenberg $X X Z$ magnet. Pisma Zh. Eksp. Teor. Fiz. 42, 414416 (1985) (translated in English by Am. Math. Soc., pp. 512-514)

35. Zamolodchikov, A.B.: Tetrahedron equations and the relativistic $\mathbf{S}$-matrix of straight-strings in 2+1-dimensions. Commun. Math. Phys. 79, 489-505 (1981)

36. Zamolodchikov, A.B.: Tetrahedron equations and integrable systems in three dimensional space. Zh. Eksp. Teor. Fiz. (Sov.) 79, 641-664 (1980)

37. Jaekel, M.T., Maillard, J.M.: Symmetry relations in exactly soluble models. J. Phys. A $\mathbf{1 5}$, 1309-1325 (1982)

38. Baxter, R.J.: Partition function of the three-dimensional Zamolodchikov model. Phys. Rev. Lett. 53, 1795-1798 (1984)

39. Baxter, R.J.: The Yang-Baxter equations and the Zamolodchikov model. Physica D 18, 321 (1986) 
40. Baxter, R.J.: On Zamolodchikov's solution of the tetrahedron equations. Commun. Math. Phys. 88, 185-205 (1983)

41. Bazhanov, V.V., Stroganov, Yu.G.: Hidden symmetry of the free fermion model. Teor. Mat. Fiz. (Sov.) 63, 417 (1985)

42. Stroganov, Yu.G.: A new calculation method for partition functions in some lattice models. Phys. Lett. 74 A, 116-119 (1979)

43. Zamolodchikov, A.B.: Factorized $\mathbf{S}$ matrices and lattice statistical systems. Sov. Sci. Rev. 2 , 1-29 (1980)

44. Jaekel, M.T., Maillard, J.M.: Inverse functional relation on the Potts model. J. Phys. A 15, 2241-2257 (1982)

45. Baxter, R.J., Forrester, P.J.: Is the Zamolodchikov model critical? J. Phys. A 18, 1483-1497 (1985)

Communicated by A. Jaffe

Received September 23, 1986; in revised form December 9, 1986 
